\title{
Nanowire-based very-high-frequency electromechanical resonator
}

\author{
A. Husain, J. Hone, Henk W. Ch. Postma, X. M. H. Huang, T. Drake, M. Barbic, \\ A. Scherer, and M. L. Roukes ${ }^{\mathrm{a})}$ \\ Departments of Physics, Applied Physics, and Electrical Engineering, California Institute of Technology, \\ Pasadena California 91125
}

(Received 8 November 2002; accepted 25 June 2003)

\begin{abstract}
Fabrication and readout of devices with progressively smaller size, ultimately down to the molecular scale, is critical for the development of very-high-frequency nanoelectromechanical systems (NEMS). Nanomaterials, such as carbon nanotubes or nanowires, offer immense prospects as active elements for these applications. We report the fabrication and measurement of a platinum nanowire resonator, $43 \mathrm{~nm}$ in diameter and $1.3 \mu \mathrm{m}$ in length. This device, among the smallest NEMS reported, has a fundamental vibration frequency of $105.3 \mathrm{MHz}$, with a quality factor of 8500 at $4 \mathrm{~K}$. Its resonant motion is transduced by a technique that is well suited to ultrasmall mechanical structures.

(C) 2003 American Institute of Physics. [DOI: 10.1063/1.1601311]
\end{abstract}

The fabrication and readout of nanometer-scale high frequency resonators has recently been a highly active area of research. Interest in these devices is motivated both by their promise as sensors and actuators, and by fundamental interest in mechanical functionality at the nanoscale. To fabricate these nanoelectromechanical systems (NEMS), researchers typically employ "top-down" techniques, i.e., highresolution lithography followed by various etching techniques to create freely suspended structures from semiconductor materials such as silicon, ${ }^{1}$ silicon carbide, ${ }^{2}$ gallium arsenide, ${ }^{3}$ and aluminum nitride. ${ }^{4}$ Advanced sensing applications require both high responsivity and ultrahigh frequency operation-among the most challenging of these are attainment of subattonewton, high frequency force sensing for magnetic resonance force microscopy, ${ }^{5}$ and the study of mechanical motion in the quantum regime. ${ }^{6}$ For future applications such as these, it is desirable to fabricate devices that are as small as possible. Top-down fabrication, however, yields device sizes limited by such factors as the resolution of electron-beam lithography, etch roughness, and the synthesis of epitaxially grown substrates. For this and other reasons, there has been significant recent interest in the integration of nanoscale materials fabricated by a "bottom-up" approach, e.g., carbon nanotubes, semiconductor, and metal nanowires. A wide variety of these nanomaterials can now be reproducibly produced, with dimensions down to the molecular scale. "Hybrid" (bottom-up+top-down) nanoelectronic devices are now widely realized from these materials by contacting them via lithographic methods; in this work we describe the successful use of this approach to make hybrid nanomechanical devices. To date, difficulties in realizing free suspension of such nanomaterials, compounded with the difficulty of detecting the minute motion of small, highimpedance structures have impeded development of hybrid NEMS. Here we describe the fabrication and readout of a very-high-frequency device consisting of a single platinum

\footnotetext{
a) Author to whom correspondence should be addressed; electronic mail: roukes@caltech.edu
}

nanowire. We also describe the simple measurement scheme employed as its readout, which is well suited to small, highimpedance NEMS devices in general.

Platinum nanowires were synthesized by electrodeposition of Pt into a commercially available nanoporous polycarbonate membrane (nominal pore diameter $20 \mathrm{~nm}$ ). ${ }^{7}$ The membrane was then dissolved in chloroform, suspending the wires in solution. This suspension was then dried, one drop at a time, upon a Si substrate that had been capped by 300$\mathrm{nm}$-thick layer of thermally grown silicon dioxide and prepatterned with $\mathrm{Au}$ alignment marks. With these, the location of the deposited wires was mapped in an optical microscope. (Optical imaging of the small wires is straightforward due to their strong light scattering properties. ${ }^{8,9}$ ) Metallic leads (5 $\mathrm{nm} \mathrm{Cr}, 50 \mathrm{~nm} \mathrm{Au}$ ) to individual nanowires were subsequently patterned by electron beam lithography, evaporation, and lift-off. Finally, the nanowires were suspended above the substrate by etching the $\mathrm{SiO}_{2}$ in hydrofluoric acid. In this step the electrode pattern also serves as a self-aligned mask, thus forming the anchor point from which the nanowires are suspended. Critical point drying completed the devices, circumventing damaging meniscus forces.

Figure 1 shows a scanning electron microscope (SEM) image of the device whose mechanical response is described later. The suspended Pt nanowire has a diameter of $43 \mathrm{~nm}$ and a length of $1.3 \mu \mathrm{m}$, with an electrical resistance at $4 \mathrm{~K}$ of $454 \Omega$. With a suspended mass of $40 \mathrm{fg}$ and a volume of $1.9 \times 10^{-15} \mathrm{~cm}^{3}$, this device is among the smallest NEMS structures whose motion has been detected directly. Modeling its response as that of an unstrained doubly clamped

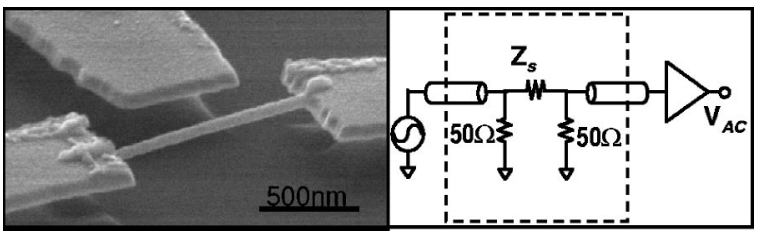

FIG. 1. (left) SEM image of the suspended nanowire device, $1.3 \mu \mathrm{m}$ long and $43 \mathrm{~nm}$ in diameter. (right) Measurement circuit used for magnetomotive drive and detection. 
beam yields a predicted fundamental mechanical resonance frequency $f_{0}$ given by ${ }^{10}$

$$
f_{0}=\frac{22.4}{2 \pi} \frac{R}{2 L^{2}} \sqrt{\frac{Y}{\rho}}
$$

where $Y$ is the Young's modulus, $\rho$ is the density, $R$ is the beam radius, and $L$ is the beam length. Using the measured dimensions and the bulk values for the Young's modulus and density of Pt (168 GPa and $21090 \mathrm{~kg} / \mathrm{m}^{3}$, respectively), Eq. (1) predicts a fundamental frequency of $64 \mathrm{MHz}$. This is lower than the measured response; possible reasons for this are described later.

Magnetomotive detection, in which an ac current drives a beam in a transverse magnetic field, has been employed to successfully actuate and read out NEMS resonators at frequencies up to and exceeding $1 \mathrm{GHz} .11,12$ On resonance the device dissipates energy from the current supply as it oscillates in the magnetic field; its behavior can be modeled as a series combination of the normal electrical resistance $R_{e}$ and a motional impedance $Z_{m}(f)$ of what appears as an RLC tank circuit. ${ }^{11}$ At the resonant frequency $f_{0}$, the magnitude of the motional impedance is given by

$$
\left|Z_{m}\left(f_{0}\right)\right| \equiv R_{m}=\frac{\xi B^{2} L^{2} Q}{2 \pi n f_{0}},
$$

where $Q$ is the quality factor, $\mathrm{m}$ the mass of the device, and $\xi$ a numerical factor that depends on the mode shape $(0.83$ for the fundamental mode of a doubly clamped beam).

Typical top-down NEMS devices have $R_{e} \sim 10-100 \Omega$ and $R_{m} \sim 1 \Omega$. In this case, the motional contribution to the overall device impedance at resonance can be relatively easily detected by measuring the reflected rf power. ${ }^{1}$ Smallerdiameter devices, such as the one measured here, will in general have much higher $R_{m}$ (which can be shown to vary as the cube of the aspect ratio), but will also possess higher $R_{e}$. Their compound effect is to make it difficult to match to $50 \Omega$ drive/detection electronics. A reflection measurement will be quite insensitive to the mechanical signal, because nearly all of the power is reflected both on and off resonance. For the present device the $454 \Omega$ electrical impedance would result in a reflectance of $81.78 \%$; an additional motional impedance of $10 \Omega$ [calculated from Eq. (2), assuming a $Q$ of $\sim 3000$ and $B=8 \mathrm{~T}$ ] would change the reflectance only to $82.14 \%$. Clearly, a transmission measurement is more appropriate for high-impedance devices.

We have employed a simple scheme, depicted in Fig. 1, to realize transmission measurements upon the device, which is located in the vacuum space of a magnet cryostat. RF power is applied at the output of a network analyzer (HP 3577A) and fed into the cryostat on coaxial cable to the device. The transmitted signal is carried to a low-noise, room temperature preamplifier providing $62 \mathrm{~dB}$ of gain via a second coaxial line. Both the drive and detection lines are terminated with $50 \Omega$ resistors next to the sample. The sample, with its effective impedance $Z_{S}(f)=R_{e}+Z_{m}(f)$, "bridges" these terminated drive and detection lines. For $Z_{s} \gg 50 \Omega$, the termination of the drive and detect lines minimizes standing waves. In this limit, the output voltage at the preamplifier is then given by high quality factors is important for technological applica-
then given by
Downloaded 18 Dec 2005 to 131.215.240.9. Redistribution subject to AlP license or copyright, see http://apl.aip.org/apl/copyright.jsp

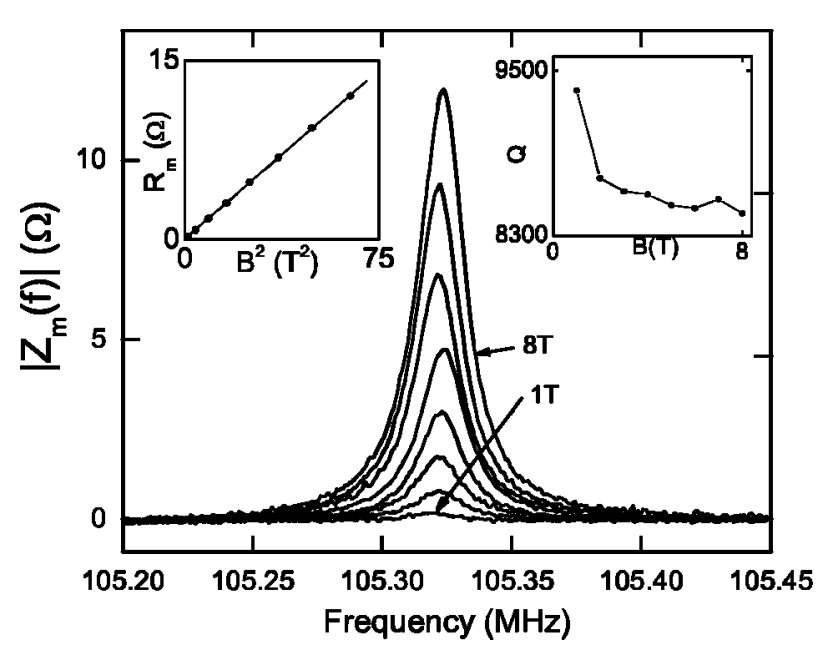

FIG. 2. Measured mechanical impedance of the Pt nanowire device as a inset shows the characteristic $\mathrm{B}^{2}$ dependence, characteristic of magnetomotive detection. The right inset shows the quality factor $Q$ as a function of magnetic field.

$$
\frac{V_{\text {out }}}{V_{\text {in }}} \approx \frac{25 \Omega}{Z_{s}+25 \Omega} \approx \frac{25 \Omega}{Z_{s}},
$$

where the factor of $25 \Omega$ is due to the $50 \Omega$ termination of the detect line in parallel with the $50 \Omega$ input impedance of the preamplifier. The electromechanical impedance $\left|Z_{m}(f)\right|$ can easily be extracted by comparing the output signal to the off-resonance background signal $V_{b}$ :

$$
\frac{\left|Z_{m}(f)\right|}{R_{e}} \cong-\frac{V_{\text {out }}-V_{b}}{V_{b}} .
$$

This scheme is of wide applicability to the readout of small-diameter, high-impedance NEMS devices because the output signal is linear in $R_{m} / R_{e}$, and because it provides broadband impedance matching, resulting in a smooth background from which it is easy to pick out a mechanical resonance peak. For extremely large device impedances, however, the cost of the approach is that it can lead to significant attenuation of the signal.

Figure 2 shows the measured motional impedance of the nanowire device, $\left|Z_{m}(f)\right|$, versus frequency. The data are obtained at $4 \mathrm{~K}$ in magnetic fields from 1 to $8 \mathrm{~T}$, with a drive signal of $100 \mu \mathrm{V} .\left|Z_{m}(f)\right|$ shows a peak at $105.3 \mathrm{MHz}$ that increases in height with increasing magnetic field, as is expected for a mechanical resonance. The measured resonance frequency is higher than the predicted frequency of $69 \mathrm{MHz}$. This is likely due to the differential thermal contraction of the beam and the substrate, which should cause the beam to be under tension. Similar effects have been seen in larger metal resonators. There is no evidence that fabrication of the nanowire by electrodeposition results in a decreased Young's modulus compared to that of bulk Pt.

The resonance curves can be fit to a Lorentzian line shape to extract values of $f_{0}, R_{m}$, and $Q$. As shown in the left inset to Fig. 2, the measured $R_{m}$ scales linearly with the square of the applied magnetic field, consistent with Eq. (2). The measured quality factor $Q$ of the device, shown in the right inset to Fig. 2, is approximately 8500, and decreases slightly with increasing magnetic field. The attainment of function of frequency, at a series of magnetic fields from 1 to $8 \mathrm{~T}$. The left 


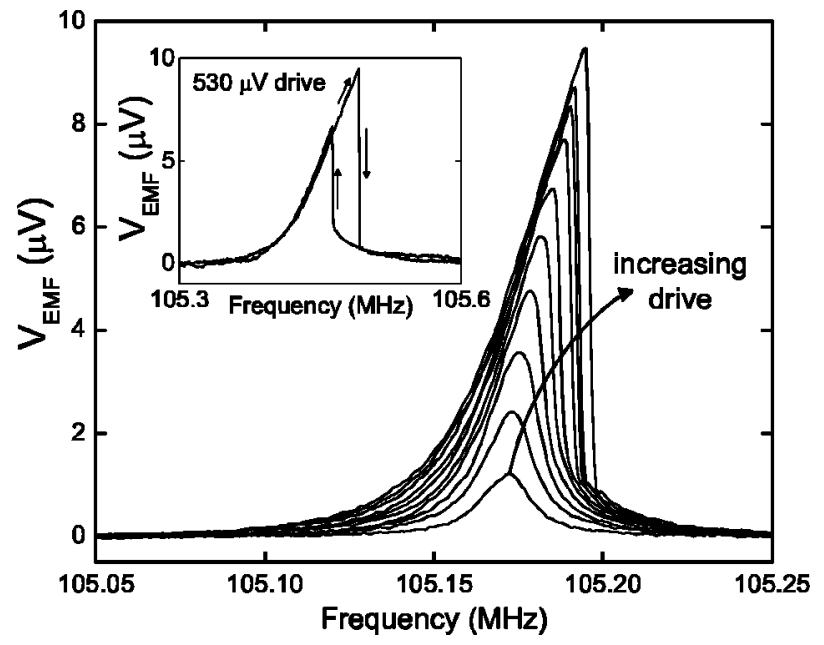

FIG. 3. Measured response of the Pt nanowire device, showing the transition to the nonlinear regime as the drive voltage is increased from 40 to 400 $\mu \mathrm{V}$, in steps of $40 \mu \mathrm{V}$. The inset shows the hysteresis visible upon sweeping up and down in frequency (note that the frequency scales are slightly different due to thermal cycling of the device).

tions of NEMS devices, but the detailed physics of energy dissipation in these devices is still not well understood. Previous studies have shown that quality factors measured for NEMS generally decrease with increasing surface areavolume ratio, apparently indicating that surface processes contribute strongly to dissipation. Carr et al., ${ }^{13}$ for example, measure the quality factors of single-crystal silicon beams and find that $Q$ decreases from $\sim 3000$ for beams with a surface area-volume ratio of $0.02 \mathrm{~nm}^{-1}$ to a value of $\sim 1000$ for devices with a ratio of $0.06 \mathrm{~nm}^{-1}$. The nanowire device described here possesses a surface area-volume ratio of $0.095 \mathrm{~nm}^{-1}$ and $Q$ of 8500 , and thus has surface-related dissipation comparable to or less than that of similar semiconductor structures.

Several important applications of NEMS resonators require extremely high force sensitivity, which, in turn requires a high compliance, low mass, and large quality factor. ${ }^{14}$ The current device is not optimized for compliance, and yields a fairly stiff effective spring constant of $\sim 2.5 \mathrm{~N} / \mathrm{m}$. Nonetheless, its force sensitivity, if limited only by thermomechanical noise (i.e., neglecting transducer and amplifier noise), is estimated to be $\sim 13 \mathrm{aN} / \sqrt{ } \mathrm{Hz}$ at $4 \mathrm{~K}$. In the future, this can be greatly enhanced by using more compliant nanowires.

Nonlinear phenomena in NEMS are of interest for a variety of applications, including mixing and parametric amplification. As they can limit dynamic range, ${ }^{15}$ they also constitute an increasingly important consideration in compliant devices with smaller dimensions, such as the one described here. Figure 3 shows the electromotive force generated by the nanowire (deduced from the measured $R_{e}, R_{m}$, and the applied drive voltage) at a series of drive amplitudes from 60 to $380 \mu \mathrm{V}$, in steps of $40 \mu \mathrm{V}$. At high oscillation amplitudes, the equation of motion for a doubly clamped beam becomes bistable, which results in discontinuities in the measured response curve. For our device, this behavior is seen at drive voltages of $260 \mu \mathrm{V}$ and above: the amplitude of oscillation increases as the frequency is swept upward, and then suddenly jumps to a lower value. The inset to Fig. 3 shows the response of the resonator as the frequency is swept both upward and downward, confirming the expected hysteretic nature of the response curve in the nonlinear regime. ${ }^{16}$

In the simplest model for the phenomenon, the critical oscillation amplitude $x_{c}$ above which bistability occurs is dependent only upon the geometry of the beam, ${ }^{17}$ and is given by

$$
x_{c}=\frac{d \sqrt{2}}{\sqrt{0.528 Q\left(1-v^{2}\right)}},
$$

where $d$ is the diameter of the beam and $v$ is the Poisson's ratio of the material. Evaluating Eq. (5) for the present device gives a critical amplitude of $0.98 \mathrm{~nm}$. As a check of our readout method, we can also compare the critical amplitude deduced from the measured signal at the onset of nonlinearity. This method gives $2.6 \mathrm{~nm}$, which is reasonable since we expect an overestimation in the second case due to losses in the signal lines. It is interesting and important to note that for even smaller and more compliant resonators, such as those made from carbon nanotubes, the critical amplitude will be in the subnanometer range. Hence, we anticipate that detecting the motion of nanowire and nanotube devices, while remaining within the linear regime, will pose a significant and generic challenge for the field of NEMS.

The authors gratefully acknowledge support from DARPA/MTO and SPAWAR via Grant No. N66001-01-X$6004 / 02-8914 / 1000000928$ and from the NSF via Grant No. ECS-0089061. H.W.Ch.P. acknowledges support, in part, from the Netherlands Organization for Scientific Research (NWO).

${ }^{1}$ A. N. Cleland and M. L. Roukes, Appl. Phys. Lett. 69, 2653 (1996).

${ }^{2}$ Y. T. Yang, K. L. Ekinci, X. M. H. Huang, L. M. Schiavone, M. L. Roukes, C. A. Zorman, and M. Mehregany, Appl. Phys. Lett. 78, 162 (2001).

${ }^{3}$ D. A. Harrington, P. Mohanty, and M. L. Roukes, Physica B 284-288, 2145 (2000)

${ }^{4}$ A. N. Cleland, M. Pophristic, and I. Ferguson, Appl. Phys. Lett. 79, 2070 (2001).

${ }^{5}$ J. A. Sidles, J. L. Garbini, K. J. Bruland, D. Rugar, O. Zuger, S. Hoen, and C. S. Yannoni, Rev. Mod. Phys. 67, 249 (1995).

${ }^{6}$ A. D. Armour, M. P. Blencowe, and K. C. Schwab, Phys. Rev. Lett. 88, 148301 (2002).

${ }^{7}$ C. R. Martin, Science 266, 1961 (1994), and references therein.

${ }^{8}$ M. Barbic, J. J. Mock, D. R. Smith, and S. Schultz, J. Appl. Phys. 91, 9341 (2002).

${ }^{9}$ J. J. Mock, S. J. Oldenburg, D. R. Smith, D. A. Schultz, and S. Schultz, Nano Lett. 2, 465 (2002).

${ }^{10}$ S. Timoshenko, D. Young, and J. W. Weaver, Vibration Problems in Engineering (Wiley, New York, 1974).

${ }^{11}$ A. N. Cleland and M. L. Roukes, Sens. Actuators A 72, 256 (1999).

${ }^{12}$ X. M. H. Huang, C. A. Zorman, M. Mehregany, and M. L. Roukes, Nature (London) 421, 496 (2003).

${ }^{13}$ D. W. Carr, S. Evoy, L. Sekaric, H. G. Craighead, and J. M. Parpia, Appl. Phys. Lett. 75, 7 (1999).

${ }^{14}$ M. L. Roukes, Phys. World 14, 25 (2001).

${ }^{15}$ M. L. Roukes, "Nanoelectromechanical Systems," Technical Digest of the 2000 Solid-State Sensor and Actuator Workshop, Hilton Head Isl, and SC, 4-8 June 2000 (Transducer Research Foundation, Cleveland, 2000), ISBN 0-9640024-3-4, http://arxiv.org/pdf/cond-mat/0008187.

${ }^{16}$ B. Yurke, D. S. Greywall, A. N. Pargellis, and P. A. Busch, Phys. Rev. A 51, 4211 (1995).

${ }^{17}$ H. A. C. Tilmans, M. Elwenspoek, and H. J. Flutiman, Sens. Actuators A 30, 35 (1992). 